\title{
On the systematics of asteroseismological mass determinations of PG 1159 stars
}

\author{
L. G. Althaus ${ }^{1,2, \star}$, A. H. Córsico ${ }^{1,2, \star}$, S. O. Kepler ${ }^{3}$, and M. M. Miller Bertolami ${ }^{1,2, \star \star}$ \\ ${ }^{1}$ Facultad de Ciencias Astronómicas y Geofísicas, Universidad Nacional de La Plata, Paseo del Bosque S/N, (1900) La Plata, \\ Argentina \\ e-mail: althaus@fcaglp.unlp.edu.ar \\ 2 Instituto de Astrofísica La Plata, IALP, CONICET-UNLP \\ 3 Instituto de Física, Universidade Federal do Rio Grande do Sul, 91501-970 Porto Alegre, RS, Brazil \\ e-mail: [acorsico;althaus;mmiller]@fcaglp.unlp.edu.ar; kepler@if.ufrgs.br
}

Received 22 August 2007 / Accepted 15 October 2007

ABSTRACT

\begin{abstract}
Aims. We analyze systematics in the asteroseismological mass determination methods in pulsating PG 1159 stars.
Methods. We compare the seismic masses resulting from the comparison of the observed mean period spacings with the usually adopted asymptotic period spacings, $\Delta \Pi_{\ell}^{\mathrm{a}}$, and the average of the computed period spacings, $\overline{\Delta \Pi_{\ell}}$. Computations are based on full PG 1159 evolutionary models with stellar masses ranging from 0.530 to $0.741 M_{\odot}$ which take into account the complete evolution of progenitor stars.

Results. We conclude that asteroseismology is a precise and powerful technique which determines the masses to a high internal accuracy, but it depends on the adopted mass determination method. In particular, we find that in the case of pulsating PG 1159 stars characterized by short pulsation periods, such as PG $2131+066$ and PG $0122+200$, the employment of the asymptotic period spacings overestimates the stellar mass by about $0.06 M_{\odot}$ as compared with inferences from the average of the period spacings. In this case, the discrepancy between asteroseismological and spectroscopical masses is markedly reduced when use is made of the mean period spacing $\overline{\Delta \Pi_{\ell}}$ instead of the asymptotic period spacing $\Delta \Pi_{\ell}^{\mathrm{a}}$.
\end{abstract}

Key words. stars: evolution - stars: interiors - stars: oscillations - stars: variables: general - white dwarfs

\section{Introduction}

Pulsating PG 1159 stars (or GW Virginis) are evolved hot stars which pose constraints to the stellar evolution theory of postasymptotic giant branch (AGB). These variable stars belong to the population of hydrogen-deficient objects characterized by surface layers rich in helium, carbon and oxygen (Werner \& Herwig 2006) which are considered the evolutionary link between post-AGB stars and most of the hydrogen-deficient white dwarfs. The origin of most PG 1159 stars is traced back to the occurrence of post-AGB thermal pulses: a born-again episode induced either by a very late thermal pulse (VLTP) experienced by a hot hydrogen-rich white dwarf during its early cooling phase - see Herwig et al. (1999), Blöcker (2001), Lawlor \& MacDonald (2003), Althaus et al. (2005), Miller Bertolami et al. (2006), or a late thermal pulse (LTP) during which hydrogen deficiency is a result of a dredge-up episode (see Blöcker 2001). During the VLTP, the convection zone driven by the helium flash reaches the hydrogen-rich envelope of the star, with the result that most of the hydrogen content is burnt.

About a third of spectroscopic PG 1159 stars exhibit multiperiodic luminosity variations with periods in the range 300$3000 \mathrm{~s}$, attributable to global nonradial $g$-modes pulsation (e.g. Quirion et al. 2007). The presence of a pulsational pattern in many PG 1159 stars has allowed researchers to infer structural

* Member of the Carrera del Investigador Científico y Tecnológico, CONICET, Argentina.

$\star \star$ Fellow of CONICET, Argentina. parameters - particularly the stellar mass - and the evolutionary status of individual pulsators - e.g. Kawaler \& Bradley (1994), Kawaler et al. (1995), O’Brien et al. (1998), Vauclair et al. (2002) and more recently Córsico \& Althaus (2006). Stellar masses of PG 1159 stars can be independently assessed by comparing the values of $\log g$ and $\log T_{\text {eff }}$, as inferred from detailed non-LTE model atmospheres (Werner et al. 1991), with tracks coming from stellar evolution modeling, i.e. the spectroscopic mass (Dreizler \& Heber 1998; Werner \& Herwig 2006). These two different approaches enable us to compare the derived stellar masses.

Recently, considerable observational and theoretical effort has been devoted to the study of some pulsating PG 1159 stars. Particularly noteworthy is the work of Fu et al. (2007) who have detected a total of 23 frequencies in PG $0122+200$ and Costa et al. (2008) who have enlarged to 198 the total number of pulsation modes in PG 1159-035, making it the star with the largest number of modes detected besides the Sun. Parallel to these observational breakthroughs, substantial progress in the theoretical modeling of PG 1159 stars has been possible (Herwig et al. 1999; Althaus et al. 2005; Lawlor \& Mac Donald 2006). In this sense, the new generation of PG 1159 evolutionary models recently developed by Miller Bertolami \& Althaus (2006) (hereinafter MA06) has proved to be valuable at deriving structural parameters of pulsating PG 1159 on the basis of individual period fits - see Córsico et al. (2007a,b), respectively, for an application to the hot pulsating RX J2117.1+3412 and the coolest member of the class, PG $0122+200$. These evolutionary models 
are derived from the complete evolutionary history of progenitor stars with different stellar masses and an elaborate treatment of the mixing and extramixing processes during the core helium burning and born again phases. The success of these models at explaining both the spread in surface chemical composition observed in PG 1159 stars and the location of the GW Vir instability strip in the $\log T_{\text {eff }}-\log g$ plane (Córsico et al. 2006) renders reliability to the inferences drawn from individual pulsating PG 1159.

As shown in MA06 the employment of detailed PG 1159 evolutionary models yields spectroscopical masses which are systematically lower - by about $0.05 M_{\odot}$ - than those derived from hydrogen-rich post-AGB tracks (Werner \& Herwig 2006). Most importantly, the resulting asteroseismological masses (as inferred from the period spacings) are usually $10 \%$ higher than the new spectroscopical masses, except for the hot pulsating PG 1159 star RX J2117.1+3412, the spectroscopical mass of which is more than $20 \%$ higher than the asteroseismological one (Córsico et al. 2007a). The mass discrepancy is a clear indication of the uncertainties weighting upon the mass determination methods, even though the spectroscopic uncertainties are of that order.

In an attempt to understand the persisting discrepancy between the asteroseismological and spectroscopical masses, Miller Bertolami \& Althaus (2007) have recently shown that previous evolution is not the dominant factor in shaping hydrogendeficient post-VLTP tracks. They conclude that the MA06 PG 1159 tracks are robust enough as to be used for spectroscopical mass determinations of PG 1159-type stars, unless opacities in the intershell region are strongly subestimated. Their results make clear that the systematic discrepancy between asteroseismological and spectroscopical masses should not be attributed to uncertainties in post-AGB tracks; rather, they call for the need of an analysis of possible systematics in the asteroseismological mass determination methods. This is precisely the core feature of the present work. Specifically, we will concentrate on the usually adopted asymptotic period spacing approach (Kawaler et al. 1995; O'Brien et al. 1998; Vauclair et al. 2002; Fu et al. 2007) used in most mass determinations of individual pulsating PG 1159 stars. The advantage of this approach lies in the fact that the mean period spacing of PG 1159 pulsators depends primarily on the stellar mass (Kawaler \& Bradley 1994; Córsico \& Althaus 2006). However, the derivation of the stellar mass using the asymptotic predictions may not be entirely reliable because they are strictly valid for chemically homogeneous stellar models, while PG 1159 stars are expected to be chemically stratified with strong chemical gradients built up during the progenitor star life. We show that this approach overestimates the seismic mass for those pulsating PG 1159 stars on the white dwarf cooling track. We also show that the discrepancy between asteroseismological and spectroscopic masses is markedly alleviated if the average of the computed period spacings, instead of the asymptotic ones, is used. In the next section, we summarize the seismological tools used to infer the stellar mass from the observed mean period spacings. We also describe the evolutionary sequences employed. In Sects. 3 and 4 we present our results and compare them with other mass determinations methods. We close the paper in Sect. 5 by summarizing our findings.

\section{Numerical tools}

The most widely used approach to infer the seismological mass of pulsating PG 1159 stars lies in the asymptotic predictions of the non-radial pulsation theory (with the notable exception of
Kawaler \& Bradley 1994 and Córsico et al. 2007a,b). In the asymptotic limit of very high radial order $k(k \gg 1$, i.e., long periods), the $g$-mode periods of a chemically homogeneous stellar model for a given degree $\ell$ and consecutive $k$ are separated by a constant period spacing $\Delta \Pi_{\ell}^{\mathrm{a}}$ given by (Tassoul et al. 1990)

$\Delta \Pi_{\ell}^{\mathrm{a}}=\frac{\Pi_{0}}{\sqrt{\ell(\ell+1)}}=\frac{2 \pi^{2}}{\sqrt{\ell(\ell+1)}}\left[\int_{r_{1}}^{r_{2}}(N / r) \mathrm{d} r\right]^{-1}$,

being $N$ the Brunt-Väisälä frequency given by

$N^{2}=-g\left[\frac{\mathrm{d} \ln \rho}{\mathrm{d} r}-\frac{1}{\Gamma_{1}} \frac{\mathrm{d} \ln P}{\mathrm{~d} r}\right]$,

where $g$ is the local gravity and $\Gamma_{1}$ the first adiabatic exponent (see Hansen \& Kawaler 1994). Note that the term in brackets is the difference between the real and the adiabatic density gradients, which determines buoyancy. The integral is taken over the $g$-mode propagation region. Note that $\Delta \Pi_{\ell}^{\mathrm{a}}$ is a function of the structural properties of the star via the Brunt-Väisälä frequency. The seismological stellar mass is constrained by directly comparing $\Delta \Pi_{\ell}^{\mathrm{a}}$ as computed from Eq. (1) with the observed mean period spacing if the effective temperature of the target star is known (by means of spectroscopy). Full advantage is taken of the fact that the $g$-mode period spacing of PG 1159 pulsators is mostly sensitive to the stellar mass and only weakly dependent on the stellar luminosity and helium-rich envelope mass fraction (Kawaler \& Bradley 1994). This feature together with the fact that no detailed pulsation calculations are required to compute $\Delta \Pi_{\ell}^{\mathrm{a}}$ turns the asymptotic period spacing into a practical tool to infer the stellar mass of pulsating PG 1159 stars.

As mentioned, the asymptotic formula given by Eq. (1) is strictly valid for chemically homogeneous stellar models and in the limit of high $k$, i.e. long periods. However, according to the current theory of stellar evolution, PG 1159 stars are expected to be chemically stratified characterized by strong chemical transitions built up during the progenitor star life. This is illustrated by Fig. 1 which displays the inner chemical abundance distribution in a typical PG 1159 star. Two main chemical transitions, emphasized with gray, are easily recognized: an inner $\mathrm{C} / \mathrm{O}$ interface left by the extra mixing episodes that ocurred during central helium burning (see Straniero et al. 2003) and an He/C/O interface that separates the helium-rich envelope from the carbon-oxygen core - modeled by nuclear processing in prior AGB and postAGB stages. Such chemical interfaces produce clear and distinctive signatures in $N$, which are critical for the mode-trapping properties of the models. These mode trapping features strongly disturb the structure of the period spectrum, thus causing the computed $g$-mode period spacing $\left(\Pi_{k+1}-\Pi_{k}\right)$ to appreciably depart from uniformity (see Kawaler \& Bradley 1994 and more recently Córsico \& Althaus 2005, 2006).

A more realistic approach to infer the stellar mass of PG 1159 stars that does not suffer from the above mentioned shortcomings is to compare the observed period spacing with the average of the computed period spacings, $\overline{\Delta \Pi_{\ell}}$. This quantity is assessed by averaging the computed forward period spacings in the same range as the observed periods, that is

$\overline{\Delta \Pi_{\ell}}=\frac{1}{n} \sum_{k} \Delta \Pi_{k}=\frac{1}{n} \sum_{k}\left(\Pi_{k+1}-\Pi_{k}\right)$

where $n$ means the number of observed modes (with $m=0$ ) of the star. In contrast with the asymptotic approach, the assessment of the asteroseismological mass via $\overline{\Delta \Pi_{\ell}}$ involves the computation of the full adiabatic period spectrum. Accurate values 


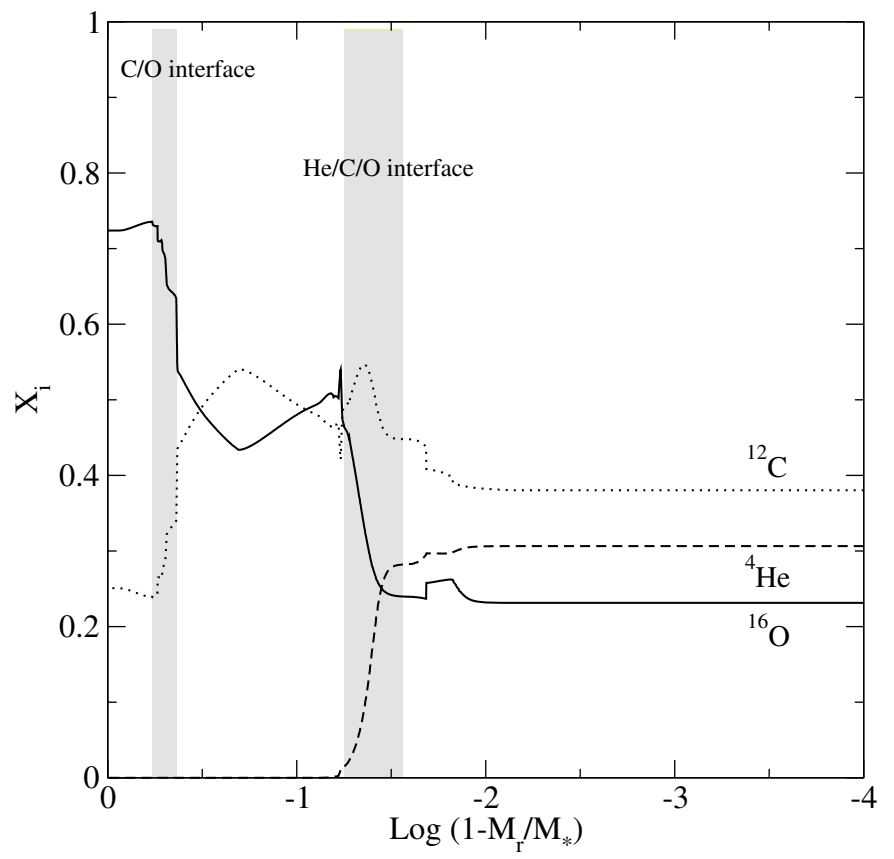

Fig. 1. The inner chemical abundance distribution corresponding to a $0.589 M_{\odot}$ PG 1159 model at $\log T_{\text {eff }}=5.18$. The approximate locations of the $\mathrm{C} / \mathrm{O}$ and $\mathrm{He} / \mathrm{C} / \mathrm{O}$ chemical transition regions are emphasized with gray.

of the adiabatic pulsation periods of pulsating PG 1159 stars requires the employment of full PG 1159 evolutionary models that reflect the thermal structure of their progenitors (Kawaler et al. 1985). In this work, we employ the evolutionary models recently developed by Althaus et al. (2005), MA06, and Córsico et al. (2006, 2007b) who computed the complete evolution of model star sequences with initial masses on the ZAMS (assuming a metallicity of $Z=0.02$ ) in the range $1-3.75 M_{\odot}$. These authors have followed all of the sequences through the thermally pulsing and mass-loss phases on the AGB to the PG 1159 regime. The evolutionary stages corresponding to the complete burning of protons shortly after the occurrence of the VLTP and the ensuing born-again episode that give rise to the H-deficient, He-, $\mathrm{C}$ - and O-rich composition characteristic of PG 1159 stars have been carefully followed for each sequence. The masses of the resulting remnants span the range $0.530-0.741 M_{\odot}$. For these PG 1159 evolutionary sequences we have computed $\ell=1, g$ mode adiabatic pulsation periods with the same numerical code and methods employed in those works (see Córsico \& Althaus, 2006 for details). In what follows, we will use these evolutionary models to compute both the mean $\overline{\Delta \Pi_{\ell}}$ and asymptotic $\Delta \Pi_{\ell}^{\mathrm{a}}$.

\section{Discrepancy between the asymptotic and the average of period spacings}

Here we employ the evolutionary models described previously to assess the asymptotic period spacing, $\Delta \Pi_{\ell}^{\mathrm{a}}$, and the average of the computed period spacings, $\overline{\Delta \Pi_{\ell}}$ as given by Eqs. (1) and (3), respectively. In Fig. 2, which summarizes the main result of our work, we show the run of these two quantities for $\ell=1$ modes (as most detected periodicities are triplets) in terms of the effective temperature for selected stellar masses. To assess the dependence of $\overline{\Delta \Pi_{\ell}}$ on the period range where the average of the period spacing is done, we compute $\overline{\Delta \Pi_{\ell}}$ for intervals of short and

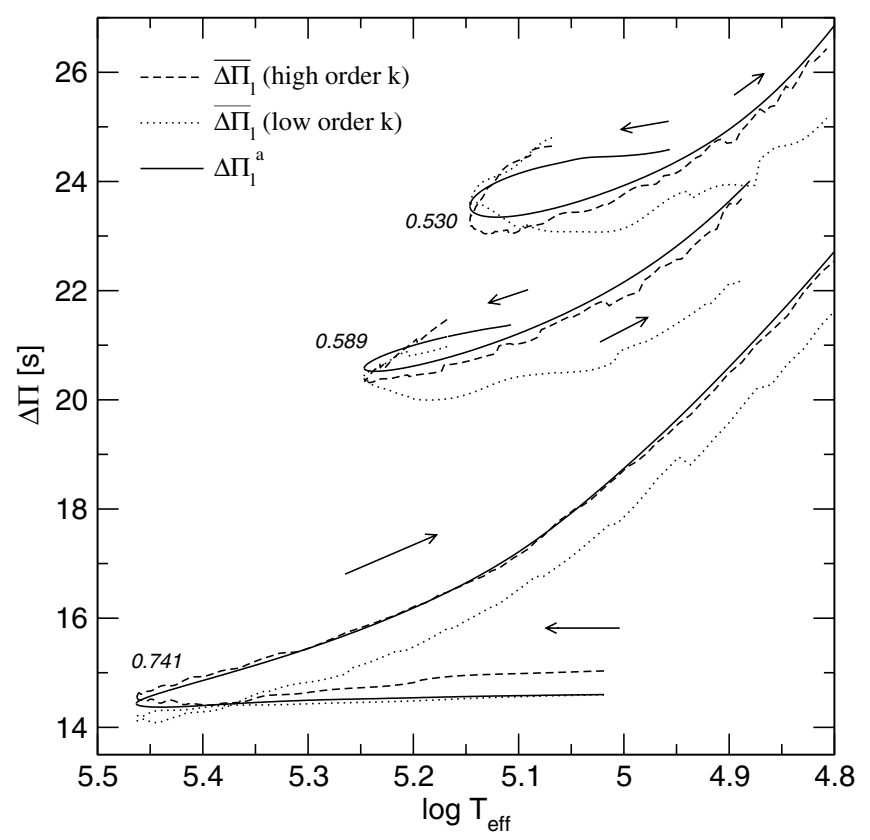

Fig. 2. The dipole $(\ell=1)$ asymptotic period spacing $\left(\Delta \Pi_{\ell}^{\mathrm{a}}\right.$, solid line $)$ is compared with the average of period spacing, $\overline{\Delta \Pi_{\ell}}$, as a function of the effective temperature for the $0.53,0.589$ and $0.741 M_{\odot}$ evolutionary sequences. For $\overline{\Delta \Pi_{\ell}}$ we consider short and long periods, i.e., low and high $k$ values (dotted and dashed lines, respectively). Stages before and after the models reach their highest effective temperature are shown. Arrows indicate the direction of evolution.

long periods (300-600 s and 900-1500 s, respectively). Different stars have different ranges. The resulting $\overline{\Delta \Pi_{\ell}}$ in each case are denoted by dotted and dashed lines. Note that both $\Delta \Pi_{\ell}^{\mathrm{a}}$ and $\overline{\Delta \Pi_{\ell}}$ decrease as the stellar mass increases. Note also that, when the star evolves along the white dwarf cooling track, the period spacings increase with decreasing effective temperature. This is due to the increasing degeneracy in the core as the star cools, causing the Brunt-Väisälä frequency to gradually decrease, and the consequent slow increment in the periods.

Most importantly, note from Fig. 2 that, generally, $\overline{\Delta \Pi_{\ell}}$ turns out to be smaller than $\Delta \Pi_{\ell}^{\mathrm{a}}$. Note also the marked dependence of $\overline{\Delta \Pi_{\ell}}$ on the period interval where it is calculated. Indeed, $\overline{\Delta \Pi_{\ell}}$ may be markedly distinct from the $\Delta \Pi_{\ell}^{\mathrm{a}}$ predictions depending on the range of periods in which the average of the period spacing is performed (or observed). This is particularly true for the evolutionary stages corresponding to the white dwarf regime, where, for a given stellar mass, $\overline{\Delta \Pi_{\ell}}$ turns out to be about $1 \mathrm{~s}$ smaller than $\Delta \Pi_{\ell}^{\mathrm{a}}$ when averages are taken on short period intervals. It is apparent that only in the case of long periods do the period spacings given by $\Delta \Pi_{\ell}^{\mathrm{a}}$ resemble those predicted by $\overline{\Delta \Pi_{\ell}}$, i.e. the asymptotic conditions are nearly reached in this case. In view of this, we expect that for those pulsating PG 1159 stars on the white dwarf cooling track, that usually exhibit short pulsation periods, the stellar mass inferred from $\overline{\Delta \Pi_{\ell}}$ becomes substantially smaller than the stellar mass determined from $\Delta \Pi_{\ell}^{\mathrm{a}}$. We address this issue in the following section. On the other hand, for the stages before the evolutionary knee, the mean $\overline{\Delta \Pi_{\ell}}$ values tend to be larger than the asymptotic $\Delta \Pi_{\ell}^{\mathrm{a}}$ ones. 


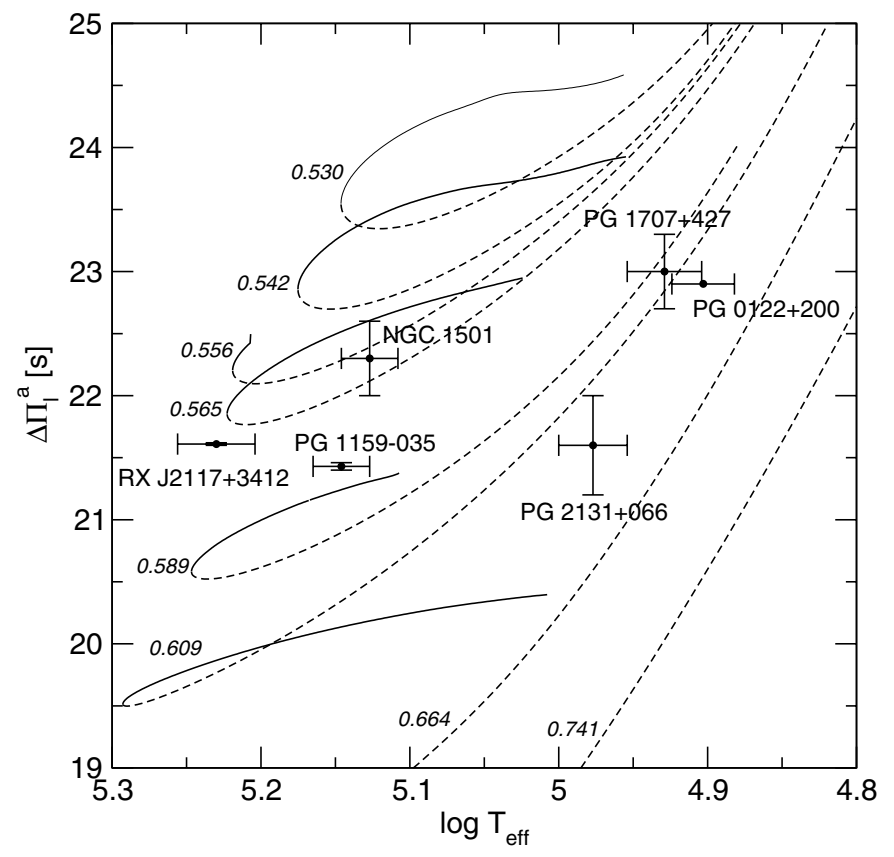

Fig. 3. The dipole $(\ell=1)$ asymptotic period spacing $\left(\Delta \Pi_{\ell}^{\mathrm{a}}\right)$ in terms of the effective temperature for various stellar masses. Solid (dashed) lines correspond to stages before (after) the models reach their highest effective temperature (evolutionary knee). Also, the location of pulsating PG 1159 stars with observed mean period spacings is shown. See Table 1 for details.

\section{Mass determinations from the observed period spacings}

Here we employ the evolutionary models described previously to infer the seismic mass of selected pulsating PG 1159 stars by comparing the asymptotic period spacing, $\Delta \Pi_{\ell}^{\mathrm{a}}$, and the average of the computed period spacings, $\overline{\Delta \Pi_{\ell}}$, with the observed mean period spacing, $\Delta \Pi^{\mathrm{O}}$. These methods allow us to infer a value of the stellar mass as long as the effective temperature of the star is determined from spectroscopy or an other method. Naturally one parameter, $\Delta \Pi$, cannot determine two properties, $T_{\text {eff }}$ and $\log g$.

In Fig. 3, we show the evolution of $\Delta \Pi_{\ell}^{\mathrm{a}}$ (for $\ell=1$ ) in terms of the effective temperature for the MA06 PG 1159 evolutionary models. The predictions corresponding to the evolutionary stages before the maximum effective temperature are indicated with solid lines, while the stages tracing the later evolution, hot white dwarf cooling branch, are denoted with dashed lines. In addition, the location of pulsating PG 1159 stars with the most recent determinations of the observed mean period spacings, $\Delta \Pi^{\mathrm{O}}$, are included in Fig. $3-$ and also listed in the sixth column of Table 1. Specifically, we include the observational data for PG 2131+066, PG 0122+200, PG 1707+427, RX J2117.1+3412, PG 1159-035, and NGC 1501. These pulsating stars are hot hydrogen-deficient, post-AGB stars for which the number of pulsation modes detected is high enough to infer an average of the period spacings. PG $2131+066$, PG 0122+200, and PG 1707+427 are evolved PG 1159 stars on the hot white dwarf cooling branch and characterized by short pulsation periods (see last column in Table 1). By contrast, RX J2117.1+3412 and NGC 1501, low-gravity and high-luminosity objects, pulsate with markedly longer periods. The pulsating NGC 1501 belongs to the [WCE] class, Wolf Rayet-type central stars of planetary nebulae with emission lines and believed to be the progenitors of PG 1159 stars.
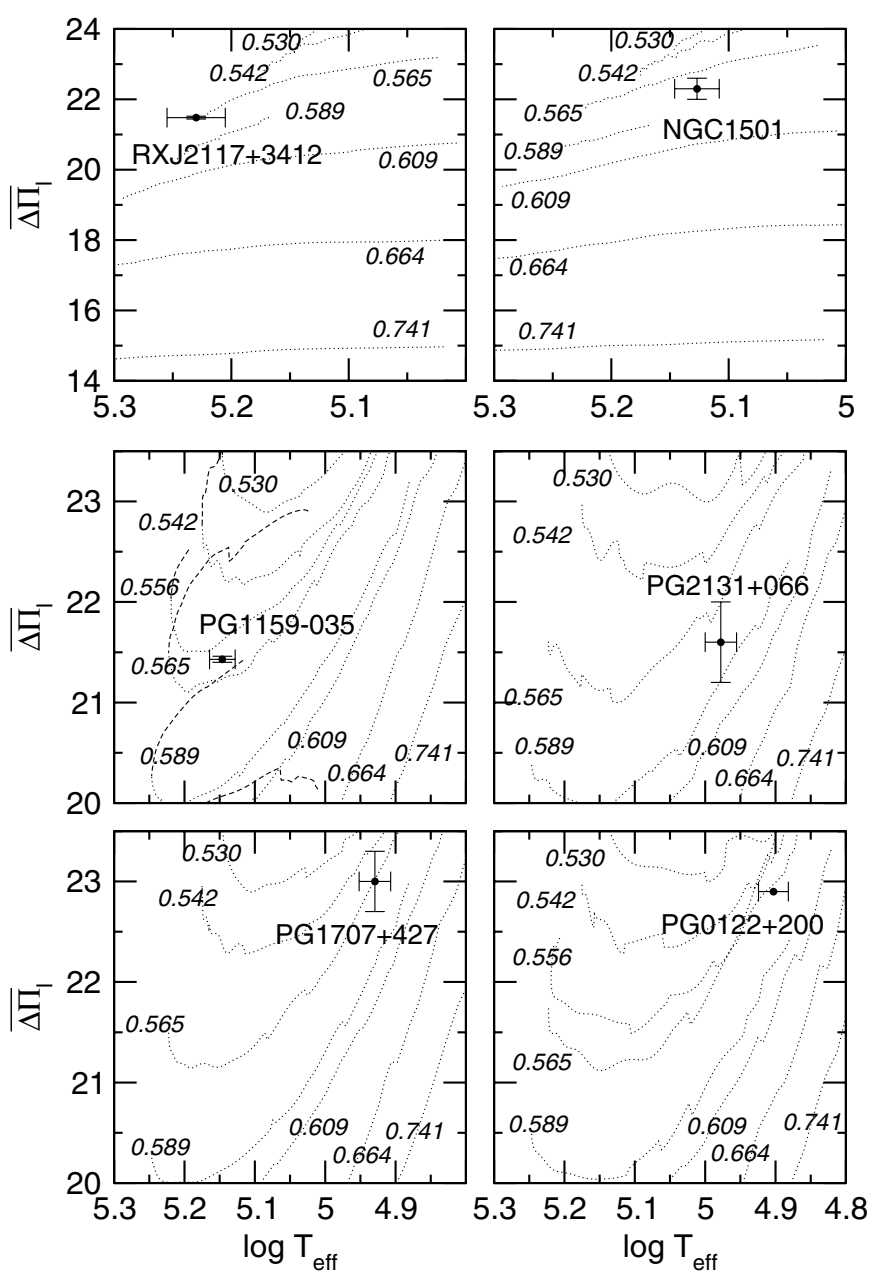

Fig. 4. The average of the computed period spacings for the PG 1159 model sequences with different stellar masses in terms of the effective temperature. Each panel corresponds to a specific pulsating PG 1159. Also, the observed mean period spacings are shown. The top two panels correspond to evolutionary stages before the sequences reach the maximum effective temperature, i.e., in the PNN stage instead of the DO stage.

From the asymptotic $\Delta \Pi_{\ell}^{\mathrm{a}}$ diagram shown in Fig. 3, the stellar mass of the above mentioned pulsating PG 1159 stars is assessed. The results are listed in the second column of Table 1. Note that the seismic masses as inferred from the use of the asymptotic approach differ by more than $10 \%$ from the spectroscopic masses (the spectroscopic masses are taken from MA06 and listed in the fifth column of Table 1$)^{1}$. This difference is particularly true for the short-period variables such as PG 0122+200, PG 2131+066, and PG 1707+427, for which the seismic mass becomes about $18 \%$ larger than the spectroscopic one.

From the discussion in the previous section, we expect smaller stellar masses for our target stars when they are derived from the mean $\overline{\Delta \Pi_{\ell}}$. This is borne out by Fig. 4, which displays $\overline{\Delta \Pi_{\ell}}$ for $\ell=1$ modes in terms of the effective temperature for different stellar masses. Each panel corresponds to a specific star, discussed above. To derive the average of the period spacings in RX J2117.1+3412 and NGC 1501, we computed $\overline{\Delta \Pi_{\ell}}$ for

1 We mention that in the case of PG 1159-035 and PG 0122+200 the uncertainty in the measured surface gravity translates into an uncertainty of $\pm 0.1 M_{\odot}$ in the spectroscopic mass (MA06). 
Table 1. Stellar masses for selected pulsating PG 1159 stars as derived from the asymptotic and the average period spacings (second and third columns). The fourth column lists the stellar mass resulting from detailed period fittings, when available. The fifth column displays the stellar mass as inferred from spectroscopy (from $T_{\text {eff }}$ and $g$ values from Werner \& Herwig 2006). The sixth column corresponds to the observed period spacings and the last column the observed range of periods for $\ell=1$. All masses are in solar units.

\begin{tabular}{ccccccc}
\hline \hline Star & $\begin{array}{c}M_{*}\left[\Delta \Pi_{\ell}^{\mathrm{a}}\right] \\
\text { This work }\end{array}$ & $M_{*}\left[\overline{\Delta \Pi_{\ell}}\right]$ & $M_{*}[$ fit $]$ & $\begin{array}{c}M_{*}[\text { spectr }] \\
\text { MA06 }\end{array}$ & $\begin{array}{c}\Delta \Pi^{\mathrm{O}} \\
{[\mathrm{s}]}\end{array}$ & $\begin{array}{c}\text { Obs. period range } \\
{[\mathrm{s}]}\end{array}$ \\
\hline PG 2131+066 & 0.627 & 0.578 & & 0.55 & $21.6^{d}$ & $339-598^{i}$ \\
PG 0122+200 & 0.625 & $0.567^{a}$ & $0.556^{a}$ & 0.53 & $22.90^{e}$ & $335-611^{e}$ \\
PG 1707+427 & 0.597 & 0.566 & & 0.53 & $23.0^{f}$ & $335-909^{f}$ \\
RX J2117.1+3412 & 0.568 & $0.560^{b}$ & $0.565^{b}$ & 0.72 & $21.62^{g}$ & $694-1530^{g}$ \\
PG 1159-035 & $0.577-0.585^{* *}$ & $0.561^{c}$ & $0.565^{c}$ & 0.54 & $21.43^{h}$ & $390-990^{h}$ \\
NGC 1501 & 0.571 & 0.576 & & 0.56 & $22.3^{j}$ & $1154-2000^{j}$ \\
\hline
\end{tabular}

References: ${ }^{a}$ Córsico et al. (2007b); ${ }^{b}$ Córsico et al. (2007a); ${ }^{c}$ Córsico et al. (2007c); ${ }^{d}$ Reed et al. (2000); ${ }^{e}$ Fu et al. (2007); ${ }^{f}$ Kawaler et al. (2004); ${ }^{g}$ Vauclair et al. (2002); ${ }^{h}$ Costa et al. (2008); ${ }^{i}$ Kawaler et al. (1995); ${ }^{j}$ Bond et al. (1996). ** The two mass values result from considering that the star is either after or before the evolutionary knee.

the high-luminosity (PNN) regime of the evolutionary sequence models, while for the remaining stars we compute values of $\overline{\Delta \Pi_{\ell}}$ for the stages following the evolutionary knee for the PG 1159 stars, i.e. the low-luminosity (DO) regime. Also for each star, the mean $\overline{\Delta \Pi_{\ell}}$ is calculated by averaging the model period spacings over the corresponding period interval in which the periodicities are indeed observed. This is the reason for the fact that the curves are different in each panel. In the third column of Table 1 we list the resulting estimation of the stellar mass for the six stars. For those pulsating PG 1159 characterized by short pulsation periods, the seismic masses as derived by this approach are appreciably lower - up to $0.06 M_{\odot}$ lower - than the values inferred by using the asymptotic period spacing. As we mentioned, this is due to the mean $\overline{\Delta \Pi_{\ell}}$ being typically $0.7-1.0 \mathrm{~s}$ smaller than the asymptotic $\Delta \Pi_{\ell}^{\mathrm{a}}$ when short periods are involved, i.e. for stages after the evolutionary knee. Thus, the discrepancy between seismic and spectroscopic masses is markedly alleviated when the average of the period spacings is used instead the asymptotic ones. Indeed, the seismic mass in this case becomes at most $6 \%$ larger than the spectroscopically derived masses, except for the hot pulsating PG 1159 star RX J2117.1+3412, the spectroscopical mass of which is more than $20 \%$ higher than the asteroseismological mass.

\section{Discussion and conclusions}

This paper explores the systematic discrepancy between spectroscopical and asteroseismological masses of pulsating PG 1159 stars. Our motivation is the result of Miller Bertolami \& Althaus (2007) that such discrepancy should not be attributed to uncertainties in post-AGB tracks, but possibly to systematics in the asteroseismological mass determination methods. Recently, Quirion has pointed out to one of us (M3B) that a possible opacity change resulting from the spread of $\mathrm{He} / \mathrm{C} / \mathrm{O}$ abundances in PG 1159 stars could be a source of uncertainty in the location of the tracks. We addressed this issue by calculating sequences in which helium and carbon are changed in the whole envelope above the helium burning shell. We find that changing helium into carbon by an amount of 0.4 by mass, shifts the track by only 0.02 dex in effective temperature (being bluer if carbon is higher). This translates into a shift of only 0.005 and $0.015 M_{\odot}$ for the spectroscopic mass near the 0.51 and $0.6 M_{\odot}$ tracks, respectively. Thus, the precise values of the $\mathrm{He} / \mathrm{C} / \mathrm{O}$ abundances do not seem to introduce appreciable changes in the masses derived by MA06.

Specifically, we have concentrated on the seismic masses that result from a comparison of the observed period spacings with the usually adopted asymptotic period spacings $\left(\Delta \Pi_{\ell}^{\mathrm{a}}\right)$ used in most mass determination of individual pulsating PG 1159 and the better suited average of the computed period spacings $\left(\overline{\Delta \Pi_{\ell}}\right)$. On the basis of full PG 1159 evolutionary models that consider the evolutionary history of progenitor stars (MA06), and the ensuing internal chemical profile, we have shown that the derivation of the stellar mass using the asymptotic period spacing is not appropriate in the case of PG 1159 stars. In particular, we demonstrate that for those pulsating PG 1159 stars characterized by short pulsation periods, i.e., the pulsating PG 1159 stars on the hot white dwarf regime (DOVs), the asymptotic $\Delta \Pi_{\ell}^{\mathrm{a}}$ differs appreciably (by more than $1 \mathrm{~s}$ ) from the mean $\overline{\Delta \Pi_{\ell}}$. Only in the case of variables with long periods (PNNVs), such as the highluminosity, log-gravity pulsating PG 1159 stars, do the $g$-mode period spacings given by asymptotic $\Delta \Pi_{\ell}^{\mathrm{a}}$ resemble those predicted by mean $\overline{\Delta \Pi_{\ell}}$. This is expected because the asymptotic conditions are approached in the limit of very high radial order $k$.

For quantitative inferences, we have computed the seismic mass resulting from the employment of the asymptotic and the average of the computed period spacing for those pulsating PG 1159 which have a sufficiently large number of detected modes to infer an observed value of the mean period spacing. Our selected stars are listed in Table 1, together with the stellar mass inferences. The employment of the asymptotic theory, in principle formally valid for chemically homogeneous stellar models at high radial index $k$, overestimates the seismic mass by about $0.06 M_{\odot}$ in the case of very short period pulsating PG 1159 stars such as PG 2131+066 and PG 0122+200. Because PG 1159 stars are expected to be chemically stratified, estimations of the stellar mass from mean $\overline{\Delta \Pi_{\ell}}$ are more realistic than those inferred by means of asymptotic $\Delta \Pi_{\ell}^{\mathrm{a}}$. Indeed, stellar masses derived from the mean $\overline{\Delta \Pi_{\ell}}$ are in good agreement with the mass values obtained from detailed period fittings. The discrepancy between asteroseismological and spectroscopical masses is markedly alleviated by the employment of the average of the computed period spacing instead of the asymptotic period spacings.

In closing, a Fortran program to derive, from our evolutionary sequences, averages of the period spacing for 
arbitrary period intervals is available at our web site http: //www . fcaglp. unlp. edu. ar/evolgroup.

Acknowledgements. We acknowledge an anonymous referee for the comments about our paper. This research was partially supported by IALP and PIP 6521 grant from CONICET.

\section{References}

Althaus, L. G., Serenelli, A. M., Panei, J. A., et al. 2005, A\&A, 435, 631

Blöcker, T. 2001, Ap\&SS, 275, 1

Bond, H. E., Kawaler, S. D., Ciardullo, R., et al. 1996, AJ, 112, 2699

Córsico, A. H., \& Althaus, L. G. 2005, A\&A, 439, L1

Córsico, A. H., \& Althaus, L. G. 2006, A\&A, 454, 863

Córsico, A. H., Althaus, L. G., \& Miller Bertolami, M. M. 2006, A\&A, 458, 259

Córsico, A. H., Althaus, L. G., Miller Bertolami, M. M., \& Werner, K. 2007a, A\&A, 461, 1095

Córsico, A. H., Miller Bertolami, M. M., Althaus, L. G., Vauclair, G., \& Werner, K. 2007b, A\&A, 475, 619

Córsico, A. H., Althaus, L. G., Kepler, S. O., Costa, J. E. S., \& Miller Bertolami, M. M. 2007c, A\&A, submitted

Costa, J. E. S., Kepler, S. O., Winget, D. E. et al. 2008, A\&A, 477, 627
Dreizler, S., \& Heber, U. 1998, A\&A, 334, 618

Fu, J.-N., Vauclair, G., Solheim, J.-E., et al. 2007, A\&A, 467, 237

Hansen, C. J., \& Kawaler, S. D. 1994, Stellar interiors (Springer-verlag)

Herwig, F., Blöcker, T., Langer, N., \& Driebe, T. 1999, A\&A, 349, L5

Kawaler, S. D., \& Bradley, P. A. 1994, ApJ, 427, 415

Kawaler, S. D., Winget, D. E., \& Hansen, C. J. 1985, ApJ, 295, 547

Kawaler, S. D., O'Brien, M. S., Clemens, J. C., et al. 1995, ApJ, 450, 350

Kawaler, S. D., Potter, E. M., Vuckovic, M., et al. 2004, A\&A, 428, 969

Lawlor, T. M., \& MacDonald, J. 2003, ApJ, 583, 913

Lawlor, T. M., \& MacDonald, J. 2006, MNRAS, 371, 263

Miller Bertolami, M. M., \& Althaus, L. G. 2006, A\&A, 454, 845 (MA06)

Miller Bertolami, M. M., \& Althaus, L. G. 2007, A\&A, 470, 675

Miller Bertolami, M. M., Althaus, L. G., Serenelli, A. M., \& Panei, J. A. 2006, A\&A, 449, 313

O'Brien, M. S., Vauclair, G., Kawaler, S. D., et al. 1998, ApJ, 495, 458

Quirion, P.-O., Fontaine, G., \& Brassard, P. 2007, ApJS, 171, 219

Reed, M. D., Kawaler, S. D., \& O'Brien, M. S. 2000, ApJ, 545, 429

Straniero, O., Domínguez, I., Imbriani, G., \& Piersanti, L. 2003, ApJ, 583, 878

Tassoul, M., Fontaine, G., \& Winget, D. E. 1990, ApJS, 72, 335

Vauclair, G., Moskalik, P., Pfeiffer, B., et al. 2002, A\&A, 381, 122

Werner, K., \& Herwig, F. 2006, PASP, 118, 183

Werner, K., Heber, U., \& Hunger, K. 1991, A\&A, 244, 437 